\title{
Development of a dual-labeled, hydrolysis probe-based, real-time quantitative PCR assay for detection of both genotypes of duck circovirus-1 (DuCV-1) and DuCV-2
}

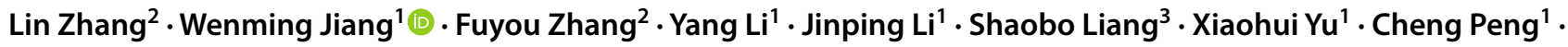 \\ Shuo Liu ${ }^{1} \cdot$ Jingjing Wang ${ }^{1} \cdot$ Shuhong Sun ${ }^{2} \cdot$ Hualei Liu ${ }^{1}$
}

Received: 5 March 2021 / Accepted: 8 July 2021 / Published online: 26 July 2021

(c) The Author(s), under exclusive licence to Springer Science+Business Media, LLC, part of Springer Nature 2021

\begin{abstract}
In this study, we developed a real-time quantitative polymerase chain reaction (qPCR) assay based on a dual-labeled hydrolysis probe to simultaneously detect both duck circovirus (DuCV) 1 and DuCV-2. The reproducibility, sensitivity and specificity of the primer set and probe were evaluated using other duck pathogens. The detection limit was 20 copies per $\mu \mathrm{L}$. The intra-assay coefficients of variation (CVs) were $\leq 0.73 \%$ and the inter-assay CVs were $\leq 1.89 \%$. No cross-reaction occurred with other duck pathogens. In addition, the qPCR assay was successfully applied to the simultaneous detection of DuCV-1 and DuCV-2 in clinical field samples. Therefore, this assay will be useful for laboratory diagnosis and epidemiological field studies of DuCV.
\end{abstract}

Keywords Duck circovirus · Dual-labeled hydrolysis probe $\cdot$ Real-time quantitative PCR $\cdot$ Simultaneous detection $\cdot$ Field samples

\section{Introduction}

Duck circovirus (DuCV), a new member of the genus Circovirus of the family Circoviridae, was first found in Mallard ducks in Germany in 2003 [1]. DuCV is a small (15-16 nm in diameter), round, nonenveloped, single-stranded DNA virus with a circular genome of approximately 1988-1996 nucleotides (nts) [2, 3]. Its genome contains three major open reading frames (ORFs), ORF1, ORF2 and ORF3 [4].

Edited by William Dundon.

Authors Lin Zhang and Wenming Jiang have contributed equally to this work.

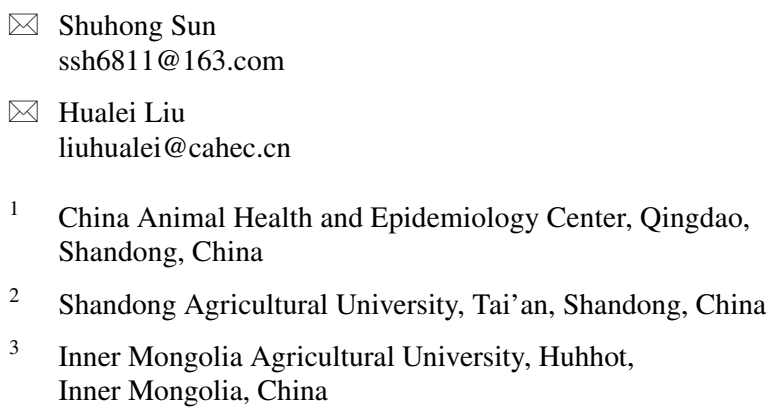

Since 2003, DuCV has been reported in a number of countries, including Hungary, China, the United States, Italy and South Korea, and in a variety of domestic duck breeds and wild ducks [5-11]. Today, DuCV is highly prevalent in the domestic duck population and is associated with considerable economic losses.

DuCV mainly affects the host immune system and causes multiple secondary infections in domestic ducks [1,11-13]. It is associated with a variety of clinical signs, such as feathering disorders, poor body condition and growth retardation, lymphocyte depletion, necrosis and histiocytosis in the bursa of Fabricius [13]. DuCV infection is often accompanied by other bacterial and viral pathogens, such as Riemerella anatipestifer, Escherichia coli, Pasteurella multocida and duck hepatitis virus [5-7, 14].

DuCV can be divided into two genotypes, DuCV-1 and DuCV-2, based on the complete genome and cap gene sequences $[11,15]$. In China, both DuCV-1 and DuCV-2 have been reported [16] and are increasingly associated with serious harm to the poultry industry $[8,11]$. Nucleotide homology between DuCV-1 and DuCV-2 is $81.8-82.5 \%$, and the conserved regions are nt $240-345$, nt $452-480$ and nt 581-678. 
Virus isolation is a fundamental diagnostic method, but no in vivo culture system is yet available for the propagation of DuCV $[17,18]$. Other diagnostic techniques, such as conventional polymerase chain reaction (PCR) [14], nested PCR [19], real-time PCR based on SYBR Green I [14, 18] and in situ hybridization (ISH), have been developed. However, these techniques are usually lengthy and require specialized equipment. For example, nested PCR requires agarose gel analysis for the detection of amplification products and has a very high risk of contamination, and ISH requires several days to complete the assay.

In this study, the development of a quantitative real-time PCR (qPCR) assay using a dual-labeled hydrolysis probe to simultaneously detect DuCV-1 and DuCV-2 is reported. The primers and probe were designed for the above mentioned conserved regions of the genome. To evaluate the developed qPCR assay for the diagnosis and monitoring of ducks with DuCV infection, we tested our method using clinical poultry samples and demonstrated that our qPCR assay could be used for the screening of virus-infected field samples.

\section{Materials and methods}

\section{Field samples and DNA extraction}

Field samples were collected from deceased ducks. DNA was isolated from a mixture of the lung, liver and spleen of each carcass using a DNeasy Blood \& Tissue kit (Qiagen, Hilden, Germany) according to the manufacturer's instructions. A total of 24 DNA samples were isolated in this study, including 20 field samples, 2 positive samples for DuCV-1 and 2 positive samples for DuCV-2. All DNA samples were stored at $-20{ }^{\circ} \mathrm{C}$ until required.

\section{Primer and probe design}

To design DuCV-1- and DuCV-2-specific primers and probes, the complete genome sequences of $130 \mathrm{DuCVs}$ from the National Center for Biotechnology Information's GenBank database (Supplementary file) were aligned using DNAStar software (DNASTAR, Madison, WI, USA) for identification of conserved regions. The following primers and probe were used: DuCVPF, 5'-TATGTTATCTTT GGGCGTGG-3' (GenBank no. MN068360, nt 457-476); DuCVPR, 5'-ACGACTACGTCATTTCCCGA-3' (nt 640-659); DuCVProbe, FAM-CACGCGGGAAGTGGT GGGACG-BHQ1 (nt 614-634).

\section{Standard plasmid construction}

To generate a DuCV standard curve for the qPCR reaction, a PCR product of $410 \mathrm{bp}$ was cloned using the primers
DuCV410F (5'-TTGAGGAGTCGCTGGGAGGA-3') and DuCV410R (5'-GACGACTACGTCATTTCCCG-3') into the vector pCloneEZ-NRS-Omni (Clone Smarter, USA) according to the manufacturer's instructions. The resulting plasmid, pDuCV410, was confirmed by DNA sequencing and purified using a Plasmid Extraction Kit (Sangon, Shanghai, China). The concentration of the pDuCV410 plasmid was determined by measuring the optical density (OD) at $260 \mathrm{~nm}$ using a Multiskan GO (Thermo Fisher, Waltham, MA, USA). The copy number of pDuCV410 was also calculated. Serial tenfold dilutions of plasmid DNA were used in amplification reactions. The dilutions were stored at $-20^{\circ} \mathrm{C}$.

\section{The qPCR analysis}

The qPCR assays were performed using an ABI QuantStudio 5 instrument (Thermo Fisher Scientific) and software (v.1.5.0). The qPCR reaction mixture contained $1 \mu \mathrm{L}$ enzyme, $10 \mu \mathrm{L} 2 \times$ AceQ qPCR Probe Master Mix (Vazyme, Nanjing, China), $1 \mu \mathrm{L}$ each primer $(0.5 \mu \mathrm{mol} / \mathrm{L}), 1 \mu \mathrm{L}$ probe $(0.25 \mu \mathrm{mol} / \mathrm{L}), 3 \mu \mathrm{L}$ DNA template and RNase-free water to a final reaction volume of $20 \mu \mathrm{L}$. The PCR thermocycling procedure was performed at $95^{\circ} \mathrm{C}$ for $5 \mathrm{~min}, 95^{\circ} \mathrm{C}$ for $10 \mathrm{~s}$ and $60^{\circ} \mathrm{C}$ for $30 \mathrm{~s}$ (fluorescence data collected) for 40 cycles. The positive reaction cycle threshold $(\mathrm{Ct})$ value was set to 35, and RNase-free water was used as a negative control.

\section{Sensitivity analysis}

To determine the limit of detection, qPCR assays were performed using serial tenfold dilutions $\left(2 \times 10^{10}\right.$ to $2 \times 10^{0}$ copies of plasmid DNA $/ \mu \mathrm{L}$ ) of the plasmid pDuCV410 as the templates. Each qPCR assay was performed in triplicate. The plasmid copy number was calculated using the URI Genomics \& Sequencing Center program.

\section{Standard curve}

The standard DuCV plasmid (pDuCV410, $2 \times 10^{8}$ to $2 \times 10^{2}$ DNA copies/ $\mu \mathrm{L}$ per sample) was used to evaluate the coefficients of variation $(\mathrm{CVs})$ of the qPCR assay. The standard curve and regression equation were established using $\mathrm{ABI}$ QuantStudio ${ }^{\mathrm{TM}}$ Design \& Analysis SE Software (v1.5.0).

\section{Reproducibility analysis}

To assess the inter-assay and intra-assay repeatability of the qPCR assay, tenfold serial dilutions of the reference materials were prepared. The average $\mathrm{Ct}$ and $\mathrm{CV}$ values were calculated according to the test results. 
For inter-assay repeatability, four tenfold serially diluted samples of the reference material were detected by qPCR three times under the same reaction conditions.

For intra-assay repeatability, four tenfold serially diluted samples of the reference material were taken, and three replicates were performed for each dilution factor. The qPCR assays were performed simultaneously.

\section{Specificity analysis}

The specificity of the assay was evaluated using other duck pathogens. Total viral DNA/RNA samples from four avian influenza viruses (H5N6, H5N8, H7N9 and H9N2 subtypes), duck coronavirus, avian paramyxovirus, new duck parvovirus, duck reovirus, duck hepatitis A virus, duck astrovirus, duck Tembusu virus and duck plague virus were assessed using the qPCR assay described in this study. Nucleic acids extracted from healthy ducks were used as negative controls.

\section{Detection of field samples}

Tissue samples from 20 deceased ducks submitted to our laboratory were tested using the qPCR assay. All DuCVpositive samples were confirmed by conventional PCR, and the Rep gene was amplified using the primer set DuCV410F and DuCV410R. Specific amplicons were purified from $1.5 \%$ agarose gels using a Gel Extraction
Kit (Sangon) and sequenced using an ABI PRISM 3730 DNA Sequencer (Thermo Fisher Scientific). Based on their sequences, all DuCV-positive field viruses were classified as DuCV-1 or DuCV-2.

\section{Results}

\section{Sensitivity of the qPCR assay}

To develop the qPCR assay, a specific primer set and probe for DuCV-1 and DuCV-2 were designed. To optimize the qPCR assay, the Rep gene plasmid was amplified and used as a quantitative standard in our system. The plasmid was serially diluted $\left(2 \times 10^{10}\right.$ to $2 \times 10^{1}$ copies per microliter $)$, and the sensitivity of our primer/probe set was determined using the qPCR assay targeting the Rep gene.

The standard curve was generated by plotting the logarithm of the plasmid copy number against the measured $\mathrm{Ct}$ values (Fig. 1). The curve had a linear correlation $\left(\mathrm{R}^{2}\right)$ of 0.998 and an efficiency of $99.35 \%$. The reaction efficiency of the assay was calculated using the slope $(-3.338)$ and the Y-intercept (36.898). We also showed that the detection limit under the qPCR conditions we used was $2 \times 10^{1}$ copies per $\mu \mathrm{L}$ (Table 1 ) with the $\mathrm{Ct}$ value cut-off for the qPCR assay set to 35 .

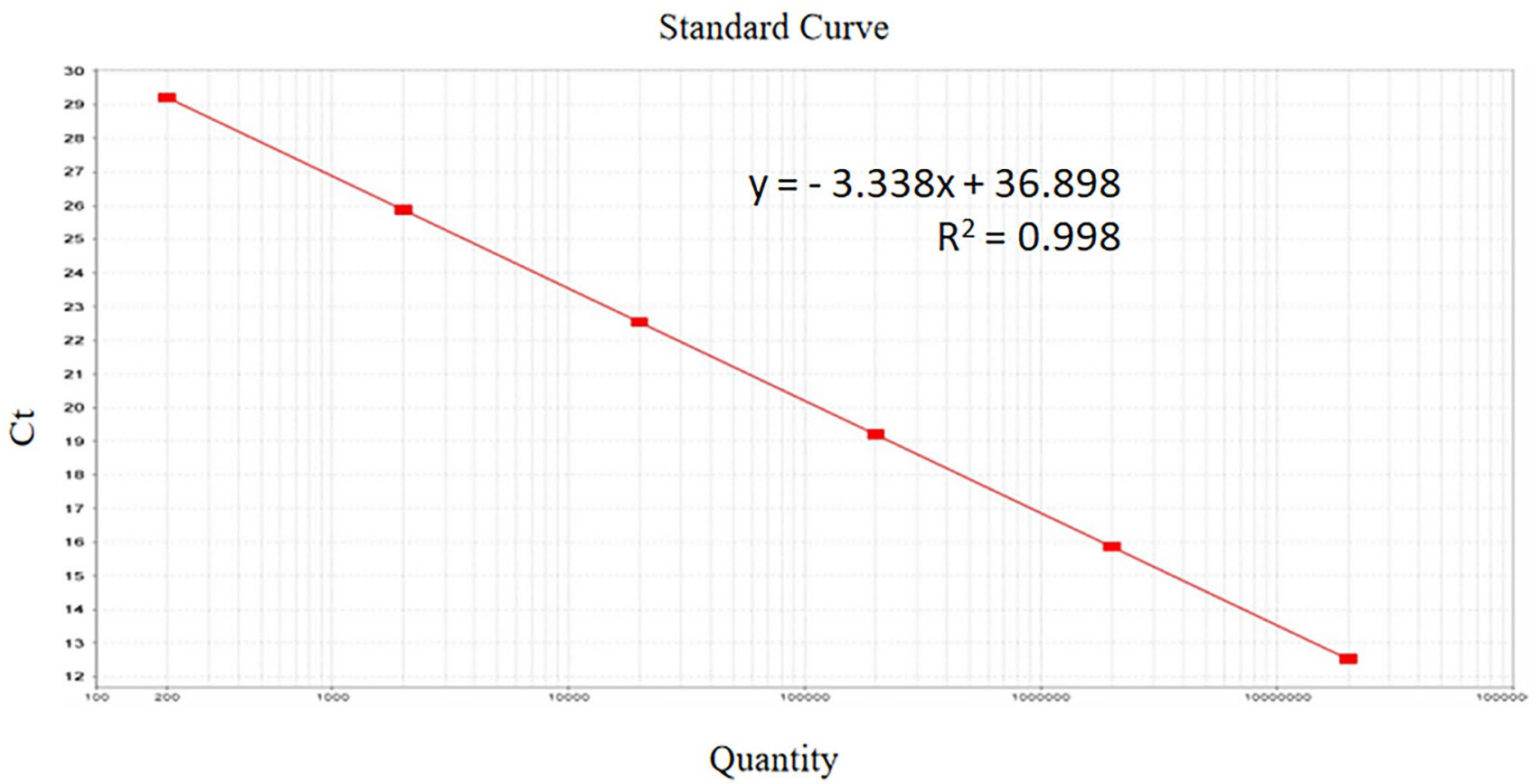

Fig. 1 Standard curve of known copies of target plasmid DNA. Reactions with copy numbers of target genes between $10^{2}$ and $10^{8}$ were used to create the standard curve by plotting fluorescence data of copy numbers against $\mathrm{Ct}$ values. The assays were in the linear range of pDuCV410 with an $\mathrm{R}^{2}$ value of 0.998 and a reaction efficiency of $99.35 \%$ 
Table 1 Amplification profile of detection for the target plasmid DNA of DuCV

\begin{tabular}{ll}
\hline Concentration of standard plasmid $($ copies $/ \mu \mathrm{L})$ & Mean $C$ t Value \\
\hline $2.0 \times 10^{10}$ & 4.69 \\
$2.0 \times 10^{9}$ & 7.91 \\
$2.0 \times 10^{8}$ & 11.01 \\
$2.0 \times 10^{7}$ & 13.29 \\
$2.0 \times 10^{6}$ & 16.98 \\
$2.0 \times 10^{5}$ & 19.97 \\
$2.0 \times 10^{4}$ & 23.26 \\
$2.0 \times 10^{3}$ & 27.18 \\
$2.0 \times 10^{2}$ & 30.71 \\
$2.0 \times 10^{1}$ & 34.20 \\
$2.0 \times 10^{0}$ & - \\
\hline
\end{tabular}

Table 2 Specific evaluation of the real-time quantitative PCR method for detection of DuCV

\begin{tabular}{lc}
\hline Tested viruses & $C$ t Value \\
\hline DuCV-1 & 13.42 \\
DuCV-2 & 18.67 \\
H5N6 & - \\
H5N8 & - \\
H7N9 & - \\
H9N2 & - \\
Duck coronavirus & 39.37 \\
Avian paramyxovirus & - \\
New duck parvovirus & - \\
Duck reovirus & - \\
Duck hepatitis A virus & - \\
Duck astrovirus & - \\
Duck Tembusu virus & - \\
Duck plague virus & - \\
\hline
\end{tabular}

\section{Specificity of the qPCR assay}

To assess the analytical specificity of the qPCR assay, different subtypes of avian influenza virus, duck coronavirus, avian paramyxovirus, new duck parvovirus, duck reovirus, duck hepatitis A virus, duck astrovirus, duck Tembusu virus and duck plague virus stored in our laboratory were tested.
As expected, the nucleic acids extracted from the DuCVpositive samples were successfully detected, whereas the viruses of other origin were not amplified by the qPCR assay (Table 2). These results showed that the qPCR assay was highly specific and exhibited no cross-reactivity for other viruses.

\section{Reproducibility of the qPCR assay}

The standard plasmid was used as the template for evaluation of the CVs of the qPCR assay. The intra- and inter-assay $\mathrm{CV}$ values ranged between $0.19 \%$ and $0.73 \%$ and $0.63 \%$ and $1.89 \%$, respectively (Table 3 ), which indicated that this method had good reproducibility.

\section{Application of the qPCR assay using field samples}

To detect DuCV in field samples using our qPCR assay, we obtained a total of 20 field samples from diseased domestic ducks in Shandong, China and isolated viral DNA from these samples. A total of six samples were confirmed to be DuCVpositive by our qPCR assay (Table 4). Next, we confirmed the sequences of all DuCV-positive samples using the universal Rep sequencing primers DuCV410F and DuCV410R. All six DuCV-positive samples belong to DuCV-1 genotype. These results showed that our qPCR assay was a promising technique for specific detection of DuCV in field samples from infected ducks.

\section{Discussion}

DuCV is a novel virus that has attracted much attention from the veterinary industry in recent years. In addition to causing death in several species of ducks along with multiple secondary infections, DuCV can also exacerbate diseases associated with multiple viruses and bacteria, as it can induce immunosuppression [7]. These mixed and secondary infections cause huge economic losses to the duck breeding industry and cannot be ignored [20].

$\mathrm{DuCV}$ has been reported in many countries, and infection rates are quite high. Prevalence rates are $46.2 \%$ in Germany [1], 84.2\% in Hungary [14], 38.2\% in Taiwan [5], 10-81.6\% in China $[12,21]$ and $21.9 \%$ in South Korea [10]. Of the
Table 3 Variance analysis of CT values quantified by realtime PCR in serially diluted standard plasmid solutions

\begin{tabular}{lcllcc}
\hline $\begin{array}{l}\text { Concentration of standard } \\
\text { plasmid }(\text { copies } / \mu \mathrm{L})\end{array}$ & \multicolumn{2}{l}{ Intra-assay variability } & & \multicolumn{2}{l}{ Inter-assay variability } \\
\cline { 2 - 3 } & $\mathrm{Ct}($ Mean $\pm \mathrm{SD})$ & $\mathrm{CV}(\%)$ & & $\mathrm{Ct}($ Mean $\pm \mathrm{SD})$ & $\mathrm{CV}(\%)$ \\
\hline $2.0 \times 10^{10}$ & $6.184 \pm 0.012$ & 0.19 & & $6.321 \pm 0.12$ & 1.89 \\
$2.0 \times 10^{9}$ & $9.212 \pm 0.054$ & 0.59 & & $9.363 \pm 0.059$ & 0.63 \\
$2.0 \times 10^{8}$ & $12.674 \pm 0.092$ & 0.73 & & $12.575 \pm 0.17$ & 1.35 \\
$2.0 \times 10^{7}$ & $16.002 \pm 0.034$ & 0.21 & & $15.873 \pm 0.12$ & 0.76 \\
\hline
\end{tabular}


Table 4 Application of the real-time quantitative PCR assay using field samples

\begin{tabular}{lll}
\hline Field samples & Ct value & $\begin{array}{l}\text { Positive } \\
(+) / \text { negative } \\
(-)\end{array}$ \\
\hline 1 & 22.40 & + \\
2 & 38.21 & - \\
3 & 39.07 & - \\
4 & 38.85 & - \\
5 & 29.50 & + \\
6 & 39.02 & - \\
7 & 38.67 & - \\
8 & 14.98 & + \\
9 & 38.53 & - \\
10 & 37.98 & - \\
11 & 38.01 & + \\
12 & 19.57 & - \\
13 & 38.82 & + \\
14 & 26.78 & - \\
15 & 39.06 & - \\
16 & 38.23 & - \\
17 & 37.89 & + \\
18 & 32.89 & - \\
19 & 38.64 & - \\
20 & 39.15 & \\
\hline
\end{tabular}

viruses two genotypes, DuCV-1 and DuCV-2 [11], there have been more reports of DuCV-1 in Germany, Hungary, the United States, China, South Korea, and Poland, but more reports of DuCV-2 in Taiwan and China.

The qPCR is a method that uses fluorescent chemicals to determine the total number of products after each round of PCR amplification. Compared with traditional PCR methods, qPCR has higher sensitivity, is simpler and takes less time [22]. In this study, a novel primer set and probe were designed for DuCV detection, and our qPCR assay was successfully employed to identify DuCV-positive field samples. To enhance the specificity of target detection, we designed a new single primer set and probe for the conserved sequences of DuCV-1 and DuCV-2. Compared with real-time PCR based on SYBR Green I, our qPCR method showed higher sensitivity [18]. Of the 20 clinical field samples, six were DuCV-positive, and the $\mathrm{Ct}$ values of these positive samples ranged from approximately 15 to 33 . Moreover, all DuCVpositive samples were sequenced and verified by GenBank BLAST processing. Our qPCR method ensured highly specific and sensitive simultaneous detection of DuCV-1 and DuCV-2. This detection platform may provide a useful screening method for clinical samples from duck farms.

Supplementary Information The online version contains supplementary material available at https://doi.org/10.1007/s11262-021-01862-9.
Author contributions LZ and WJ designed the study. FZ, YL, JL, SL, $\mathrm{XY}, \mathrm{CP}$ and SL carried out the experiments, collected the data and participated in drafting the manuscript. LZ drafted the manuscript, which underwent revisions by WJ and SS. All authors read and approved the final manuscript.

Funding This work was funded by the Innovation Fund of the China Animal Health and Epidemiology Center.

Data availability Please contact the author (Dr. Jiang) for data requests.

\section{Declarations}

Conflict of interest The authors declare that they have no conflict of interest.

Ethical approval Not required.

Consent for publication All authors consent for publication.

\section{References}

1. Hattermann K, Schmitt C, Soike D, Mankertz A (2003) Cloning and sequencing of Duck circovirus (DuCV). Adv Virol 148:2471-2480

2. Todd D (2004) Avian circovirus diseases: lessons for the study of PMWS. Vet Microbiol 98:169-174

3. Hughes AL, Piontkivska H (2008) Infection genetics and evolution. J Mol Epidemiol Evol Genet Infect Dis 8:130-138

4. Xiang QW, Wang X, Xie ZJ, Sun YN, Zhu YL, Wang SJ, Liu HJ, Jiang SJ (2012) ORF3 of duck circovirus: a novel protein with apoptotic activity. Vet Microbiol 159:251-256

5. Chen CL, Wang PX, Lee MS, Shien JH, Shien HK, Ou SJ, Chen $\mathrm{CH}$, Chang PC (2006) Development of a polymerase chain reaction procedure for detection and differentiation of duck and goose circovirus. Avian Dis 50:92-95

6. Banda A, Galloway-Haskins RI, Sandhu TS, Schat KA (2007) Genetic analysis of a duck circovirus detected in commercial pekin ducks in New York. Avian Dis 51:90-95

7. Zhang X, Jiang S, Wu J, Zhao Q, Sun Y, Kong Y, Li X, Yao $M$, Chai T (2009) An investigation of duck circovirus and coinfection in Cherry Valley ducks in Shandong Province, China. Vet Microbiol 133:252-256

8. Wan CH, Fu GH, Shi SH, Cheng LF, Chen HM, Peng CX, Lin S, Huang Y (2011) Epidemiological investigation and genome analysis of duck circovirus in Southern China. Virol Sin 26:289-296

9. Matczuk AK, Krawiec M, Wieliczko A (2015) A new duck circovirus sequence, detected in velvet scoter (Melanitta fusca) supports great diversity among this species of virus. Virology Journal $12: 121$

10. Cha SY, Kang M, Cho JG, Jang HK (2013) Genetic analysis of duck circovirus in Pekin ducks from South Korea. Poult Sci 92:2886-2891

11. Zhang Z, Jia R, Lu Y, Wang M, Zhu D, Chen S, Yin Z, Chen X, Cheng A (2013) Identification, genotyping, and molecular evolution analysis of duck circovirus. Gene 529:288-295

12. Wang D, Xie X, Zhang D, Ma G, Wang X, Zhang D (2011) Detection of duck circovirus in China: a proposal on genotype classification. Vet Microbiol 147:410-415

13. Soike D, Albrecht K, Hattermann K, Schmitt C, Mankertz A (2004) Novel circovirus in mulard ducks with developmental and feathering disorders. Vet Rec 154:792-793 
14. Fringuelli E, Scott AN, Beckett A, McKillen J, Smyth JA, Palya V, Glavits R, Ivanics E, Mankertz A, Franciosini MP, Todd D (2005) Diagnosis of duck circovirus infections by conventional and real-time polymerase chain reaction tests. Avian Pathol J WVPA 34:495-500

15. Wen H, Wu Y, Yang C, Zhang X, Lian C, Chen H, Han L (2014) Comments on duck circovirus (DuCV) genotype definition. Gene 538:207-208

16. Niu X, Liu L, Han C, Li J, Zeng X (2018) First findings of duck circovirus in migrating wild ducks in China. Vet Microbiol 216:67-71

17. Liu SN, Zhang XX, Zou JF, Xie ZJ, Zhu YL, Zhao Q, Zhou EM, Jiang SJ (2010) Development of an indirect ELISA for the detection of duck circovirus infection in duck flocks. Vet Microbiol 145:41-46

18. Wan C, Huang Y, Cheng L, Fu G, Shi SH, Chen H, Peng C, Lin F, Lin J (2011) The development of a rapid SYBR Green I-based quantitative PCR for detection of duck circovirus. Virol J 8:465

19. Halami MY, Nieper H, Muller H, Johne R (2008) Detection of a novel circovirus in mute swans (Cygnus olor) by using nested broad-spectrum PCR. Virus Res 132:208-212
20. Hong YT, Kang M, Jang HK (2018) Pathogenesis of duck circovirus genotype 1 in experimentally infected Pekin ducks. Poult Sci 97:3050-3057

21. Liu H, Li LX, Sun WC, Shi N, Sun XT, Jin NY, Si XK (2020) Molecular survey of duck circovirus infection in poultry in southern and southwestern China during 2018 and 2019. BMC Vet Res $16: 80$

22. Souza FA, Dos Santos Junior EM, Laguardia-Nascimento M, Freitas TRP, Damaso CR, Rivetti Junior AV, Camargos MF, Fonseca Junior AA (2019) Validation of a real-time PCR assay for detection of swinepox virus. Arch Virol 164:3059-3063

Publisher's Note Springer Nature remains neutral with regard to jurisdictional claims in published maps and institutional affiliations. 